\title{
De-novo draft genome sequence of Crocus Sativus L, Saffron, a golden condiment.
}

$5 \quad{ }^{1}$ National Center for Biological Sciences, Bellary Road, Bangalore 560065

$6 \quad{ }^{2}$ School of Biotechnology, University of Jammu, J\&K, India, 180006

7 *Author for correspondance: Prof. Ramanathan Sowdhamini, mini@ncbs.res.in

9 Abstract:

10 Crocus sativus L, saffron is the highest priced but low yielding plant of medicinal and culinary im11 portance. Despite its economic status, the omic information on this plant is very scarce, with only a 12 couple of transcriptomics and epigenetic studies. In the present study, the draft genome sequence of 13 C. sativus has been assembled using Illumina sequencing. In fact, this is the first genome sequence 14 from any member of family Iridaceae. Genome size of $C$. sativus was estimated to be $3.5 \mathrm{~Gb}$ and 15 the draft genome is $3.01 \mathrm{~Gb}$ long with $84.24 \%$ genome coverage. In total, 8,62,275 repeats and $169,64,231$ SSR markers have been identified. A total of 53,546 functional genes were annotated, out of which, 43,649 proteins were associated with GO annotation. 5726 proteins were identified as transcription factors, with MYB \& MYB related family proteins being more abundant. Orthology analysis of $C$. sativus with 3 different monocot species of the same plant order and rice (model monocot plant) revealed 7328 proteins clusters to be conserved in all the five plant species, whereas 2510 proteins cluster were unique to $C$. sativus only. 10,912 unigenes of $C$. sativus were mapped to 387 KEGG pathways of monocot. The genes involved in the pathway of apocarotenoids biosynthesis (crocin, crocetin, picrocrocin, and safranal) were present in the draft genome.

24 Keywords: Crocus sativus, denovo genome assembly, apocarotene biosynthesis pathway, MYB Tfs, SSR markers, Orthology analysis.

\section{Introduction:}

Plant genomics, with the increasing number of whole genome sequences available, has unlocked the genetic treasures, that would be impossible in absense of the genome sequence. Though second and third generation sequencing technologies, coupled with ever advancing bioinformatic tools/pipelines, have made the sequencing of complex and huge genomes economical and easy, but still till date there are only approximately 1565 plant genome sequenes available in databanks 
34 plant genome are rice (Choi et al., 2020), Maize (Liu et al., 2020), Asparagus (Harkess et al., 2017), Wheat (Alonge et al., 2020) and tea (Xia et al., 2020) etc., however the genome of the plants belonging to Crocus genera or Iridaceae family, have not reported so far.

Saffron (C. sativus) referred as 'Golden Condiment' is world's most expensive spice costing about 70,000 INR/pound, with medicinal properties and cosmetic uses (Mzabri et al., 2019; Magotra et al., 2021). More than 150 volatile and aroma-yielding compounds contribute to the flavor, color, and aroma of the Saffron spice, wherein the main chemical constituents in the stigma of saffron are crocin, crocetin, picrocrocin, and safranal (Samarghandian et al., 2014; Maggi et al., 2020). $C$. sativus is an autumn-flowering perennial sterile triploid plant $(2 \mathrm{n}=24)$ with, $\sim 3.5 \mathrm{~Gb}$ haploid genome (Brandizzi and Caiola 1996, 1998). Being sterile, it fails to produce viable seeds and reproduces vegetatively by underground corms and is reported to lack genetic variation. Various molecular markers (RAPD, ISSR, AFLP, SSR microsatellites) and epigenetic approaches have suggested the existence of limited genetic variability (Rubio-Moraga et al., 2009; Siracusa et al., 2013; Busconi et al., 2018; Mir et al., 2021). To discover authentic genetic markers, mining genes for secondary metabolites and improvement of breeding, sequencing of its genome was the only alternative. In addition, it's ancestry is also controversial (Alsayied et al., 2015; Nemati et al., 2019), that could be also settled, if its complete genome sequence is available.

Hybrid sequencing approaches, comprising of second and third generation sequencing technologies, have facilitated sequencing of complex genomes economically. Illumina sequencing technology is preffered for first sequencing attempt, as it generates good sequencing data for better genome coverage and has low error rate as compared to third generation sequencing technologies (Edwards and Batley 2010). In the present study, C. sativus de novo draft genome sequence has been assembled using short read Illumina sequencing technology. The draft genome sequence unravels the putative markers, metabolic pathways, transcription factors and orthologous genes.

Materials and Methods:

Sample collection, genome size estimation, whole genome library preparation and Sequencing: C. sativus corms were collected from Kishtwar, J\&K $\left(33.3116^{\circ} \mathrm{N}, 75.7662^{\circ} \mathrm{E}\right)$ in 2019 . Corms $^{\circ}$ were grown in the pots for period of three months and leaves were harvested for genome size estimation. Genome size of the plant was estimated by flow cytometric (Hare and Johnston 2011) and k-mer based method using JellyFish (Marçais and kingsford 2011). Genomic DNA was extracted from corm tissue using CTAB method (Rogers and Bendich 1994) and quality and quantity was accessed using Qubit (Invitrogen) and agarose gel electrophoresis. 3 ug DNA was used to construct WGS DNA libraries with 550bp and 800bp insert sizes using NEB next Ultra DNA Library Prepa- 
tation (Agilent 4200) and Qubit HS DNA Assay Kit (Invitrogen). Sequencing was done on the HiSeqX platform (150-bp paired-end (PE) reads) to generate $321 \mathrm{~Gb}$ data ( $\sim 92 \mathrm{X}$ coverage).

De-novo Genome Assembly and statistics: Quality of raw reads was evaluated using FastQC tool

(Andrews S. 2010) and low quality bases (<q30) and sequencing adapters were removed using trimmomatic software (Bolger et al., 2014). De-novo genome assembly was performed using two assemblers namely Soapdenovo2 (Luo et al., 2012) and MaSuRCA (Zimin et al., 2013). Soapdenovo2 assembly was executed using different kmers (73 kmer predicted by KmerGenei along with 69, 71 kmers) (Chikhi and Madvedev 2013). The statistics of soapdenovo2 assemblies were compared to select the better assembly that was designated as Cs_Assembly_1. MaSuRCA assembly was done using the raw reads and was designated as Cs_Assembly_2. The quality of assemblies was accessed using BUSCO against Viridiplantae lineage from OrthoDB database (Simao et al., 2015). Subsequently, raw illumina reads were mapped back to Cs_Assembly_2 using Bowtie2 (Langmead 2010) and previously published transcriptome data (Baba et al., 2015; Jain et al., 2016) and mapped to Cs_Assembly_2 using BWA (Li and Durbin 2010). Repetitive regions in Cs_Assembly_2 was identified using Repeatmasker and GenomeScope v2 (Ranallo-Benavidez et al., 2020, Smit et al., 2015) and SSR markers were identified using MISA (Beier et al., 2017).

Genome prediction and annotations, orthology and metabolic pathway analysis: Cs_Assem-

bly_2 was further analysed for gene prediction using the MAKER (Campbell et al., 2014) wherein

C. sativus transcriptome data was used as EST evidence (Jain and coworker 2016), Viridiplantae database (UNIPROT) as protein evidence, maize as Augustus gene prediction model and Oryza sativa as snap hmm. Predicted proteins were further annotated using BLASTp against NR (NCBI) and viridiplanteae (UNIPROT) database with modified parameters (E-value-1 $\mathrm{e}^{-3}$, sequence identity $>40 \%$ and query coverage $>70 \%$ ). Annotated proteins were analysed for GO annotations against biological processes, cellular component and metabolic processes using WEGO (Jia et al., 2018). Transcription factors (Tfs) proteins were identified against PlantTFDB (Jinpu et al.,2017) using BLASTp with the modified parameters $\left(\mathrm{E}-\mathrm{value}-1 \mathrm{e}^{-3}\right.$, sequence identity $>30 \%$, query coverage $>70 \%$ ). Orthologous genes were compared with Asparagus officinalis, Dendrobium catenatum, Phalaenopsis equestris, Apostatia shenzhenica of the same plant order along with Oryza sativus (Rice) using Orthovenn 2 (Xu et al., 2019) The proteins sequences of all the plants were downloaded from Phytozome database (David et al., 2012). Various metabolic pathways in C. sativus genome were analysed using KAAS webserver (Kyoto Encyclopedia of Genes and Genomes Automatic Annotation Server) (Moriya et al., 2007).

101 Data availability: Whole genome sequencing raw reads has been submitted to NCBI SRA under

102 bioproject PRJNA734464. The draft genome of Crocus sativus has been submitted in SRA under 
103 bioproject PRJNA739096. All the processed data including draft genome, annotated proteins, SSR,

104 Transcription factors, and supplementary tables and figures can be accessed at http://caps.ncb105 s.res.in/download/csat.

106

107

108

109

110

111

112

113

\section{Results and Discussion}

Crocus sativus genome, being reported here, is the first draft genome sequence of the plant belonging to the Iridaceae family. Genome size of $C$. sativus was estimated to be $3.5 \mathrm{~Gb}(3,578,575,507$ bases), using flow cytometry and kmer method. Genome size estimated was comparable to earlier reports, wherein it was estimated to be $3.44 \mathrm{~Gb}$ using flow cytometry being grown in Italy, Spain and Israel (Brandizzi and Cajola 1997; 1998). On the basis of size of the genome, $321 \mathrm{~Gb}$ WGS data of C. sativus was generated, with an overall coverage of $\sim 92 \mathrm{X}$ using Illumina sequencing (Table 1). De novo genome assembly and annotation of $C$. sativus was performed using the bioinformatics pipeline represented in Fig 1 for easy comprehension.

De-novo genome assembly: C. sativus genome was de-novo assembled using SOAPdenovo2 (Luo et al., 2012) and MaSuRCA softwares (Zimin et al., 2013). Soapdenovo2 assembly with kmer 71 was comparatively better than other two kmers (69 and 73) and was designated as Cs_Assembly_1 with N50 value of 1596 and 77.9\% genome coverage (Table 2). De-novo assembly with MaSuRCA was designated as Cs_Assembly_2 with N50 value of 1860 and 84.24\% genome coverage. Cs_Assembly_2 was found comparatively better than Cs_Assembly_1 as the assembly statistics, such as N50, largest scaffold, genome coverage and BUSCO completeness were higher in Cs_Assembly_2 than Cs_Assembly_1. (Table 2). Further, $\sim 87.28 \%$ of raw reads mapped back to Cs_Assembly_2, thereby indicating that most of data has been utilized for genome assembly. In addition, two previously published transcriptome data sets (Jain et al., 2016; Baba et al., 2015) were mapped to the Cs_Assembly_2 and mapping percentage of 99.92\% and 92.02\% were observed against Cs_Assembly_2 (Table 3). High mapping percentage represented the presence of most of the reported exons/ CDS in the Cs_assembly_2 even though the genome assembly was fragmented with less N50 value. Polygonum cuspidatum genome was de-novo assembled using Soapdenovo2 with Illumina reads and generated an assembly of $2.56 \mathrm{~Gb}$, with N50 value of 3215 and $98.5 \%$ genome coverage (Zhang et al., 2019). Similarly, the genome of Linum usitatissimum, flax plant was de novo assembled using Illumina reads having N50 scaffold of $694 \mathrm{~Kb}$ with $81 \%$ of genome coverage (Wang et al., 2012). Genome coverage of $C$. sativus was comparatively more than flax genome but less than Polygonum cuspidatum genome using same sequencing technologies.

Repeats and SSR markers: Total repeats length in C. sativus genome (Cs_assembly_2 ) was $1,460,908,750$ bp $(40.8 \%)$ as predicted by GenomeScope version 2. A total of 8,62,275 repeats were identified in Cs_assembly_2 wherein simple repeat (48.41\%) and LTR (30.34\%) were the most abundant in the genome. Specifically, Copia \& Gypsy were the most abundant LTR repeats 
138 (Table 4). A total of 9,64,231 SSR markers were identified in Cs_assembly_2 wherein monomeric 139 SSR repeats $(4,86,140-50.4 \%)$ were more abundant as compared to dinucleotide $(2,94,819$ $14030.5 \%)$ and trinucleotide repeats $(1,46,991-15.2 \%)$ with "A", "TA" "TTG" most abundant SSRs in 141 each groups. The abundance of Tetranucleotide (15,375-1.59\%), pentanucleotide (8596-0.9\%) and 142 hexanucleotides (12,310-1.27\%) repeats each was less than $2 \%$ of total SSRs with "AAAT", 143 "TATAT" and "TAACCC" most abundant in respective SSRs (Table 5). SSR markers are reported to 144 be multi-allelic, relatively abundant, widely dispersed across the genome and have been used in genetic diversity analysis, parentage assessment, species identification and mapping genetic linkage (Feng et al., 2016). These markers can be further evaluated for their application in C. sativus. Earlier studies on C. sativus transcriptome has reported the presence of 16,721 SSRs (Jain et al., 2016) and 79,028 SSRs (Qian et al., 2019) using transcriptome analysis, but higher number of SSR $(9,64,231)$ were discovered in the present study based on genome sequence.

Gene prediction and annotation: In total 2,54,038 proteins were predicted from Cs_assembly_2 using MAKER pipeline. A total of 52,435 and 52,545 proteins were annotated based on BLASTp against NR and viridiplanteae database respectively (Table 6). BUSCO analysis revealed the presence of $75.7 \%$ of the plant conserved genes/orthologues in the C. Sativus genome. Out of total proteins, 51\% (26796) were annotated to 8 top-hit plant species (Fig 2). Maximum number of proteins were annotated against Asparagus officinalis (9213) indicating C. sativus to be phylogenetically closer to Asparagus officinalis, as both the plants belong to same plant order Asparagales (Fig 2). $85 \%$ of total proteins $(43,649)$ were associated with gene ontology (GO) ids and classified into biological processes (BP: 22,092 proteins) abundant in cellular and metabolic processes, cellular components (CC: 24,399 proteins) mostly localised in cell and organelle parts and molecular functions (MF: 34,442 proteins) most abundant in catalytic and transporter activities (Table S1).

Transcription factors: Out of the total annotated proteins, 5726 unique C. sativus proteins were identified as transcription factors (TFs) belonging to 57 TFs families. MYB \& MYB related family proteins (11.86\%), being more abundant Tfs, followed by bHLH, C2H2, NAC, FAR1, C3H, ERF, bZIP, WRKY and B3 were the top 10 abundant transcription factors family proteins (Fig 3, Table S2). TFs like MYB \& MYB related, bHLH, WRKY are reported to regulate secondary metabolite (apocarotenoid) biosynthesis in C. sativus (Jain et al., 2016). Earlier reports on C. sativus transcriptome has identified less number of TFs $(3819$, 2601), whereas the most abundant Tfs family remains same (Jain et al., 2016; Baba et al., 2015).

169 Comparative genomics: $C$. sativus annotated proteins $(52,545)$ was compared with 3 monocots 170 plants of same order, whose genome and annotations were available in Phytozome database (David 
172 with a model monocot plant Oryza sativus (Rice) using Orthovenn2. A total of 23,744 proteins clus-

173 ter were found in all the plants wherein 21,606 were orthologous clusters that were atleast common

174 in two species and 2138 were single copy gene clusters wherein each cluster have only one gene 175 from each plant species. Conserveration of 7328 proteins clusters, comprising of 51,803 proteins, 176 was observed among the five species (C. sativus:10,001 proteins, A. officinalis:9552, $P$. equestris:9012, A. shenzhenica: 8570 and O. sativa:14,668) (Fig 4a and 4b). The conserved proteins clusters were found to be associated with biological processes (BP-23,010 proteins), cellular component (CC-582 proteins) and molecular functions (MF-957 proteins) and were enriched in defence response, RNA modification, DNA integration, regulation of transcription, rRNA processing and protein phosphorylation (Supplementary table S3). However, 2510 protein clusters (7914 proteins) were unique to Crocus sativus only, out of which 1636 clusters (4595 proteins) were associated with slimmed GO terms (BP: 5201, CC: 63, MF:303 proteins) associated with nucleic acid binding, transferase, hydrolase, oxidoreductase activity and protein and DNA binding activity (Supplemenatary Table S3). As per orthology analysis also, C. sativus was found phylogenetically closer to $A$. officinalis as more protein clusters were orthologous between Crocus sativus and Asparagus officinalis than to other plants compared in the study (Fig 5).

Metabolic pathway analysis: A total of 10,912 C. sativus proteins were mapped to 395 KEGG pathways of monocots. Various pathways like carbohydrate metabolism, energy metabolism, lipid metabolism, nucleotide metabolism, amino acid metabolism, glycan metabolism, metabolism of cofactors and vitamins along with biosynthesis of terpenoids, polyketides and other secondary metabolites were found complete wherein all the genes involved in pathway were present in draft assembly (Table S4). We, further, investigated the presence of genes involved in the synthesis of apocarotenoids namely crocins, picrocrocin, and safranal that are produced in the stigma of C. Sativus. These apocarotenoids impart red color, bitter taste, and pungent aroma to stigma of saffron and have various medicinal properties (Maggi et al., 2020). The molecular basis of apocarotenoid biosynthesis in C. Sativus has been well studied using transcriptomics studies (Baba et al., 2015; Jain et al., 2016). In the present study, the genes encoding the enzymes involved in carotene biosynthesis pathway, regulating the apocarotenoids synthesis, were present in the C.sativus genome (Fig 6). This is the first de novo draft genome sequence of Crocus sativus that needs to be complemented with the long read sequencing technology (PacBio) to fill in the gaps in the present genome to generate a complete genome sequence. However this draft genome sequence, in addition to revealing previous

203 unknown genomic information on saffron, will also be used as a reference genome for future genome sequencing attempts in saffron. 
Authors are grateful to Mr. Shanu Magotra, School of Biotechnology, University of Jammu, J\&K for his help in sample collection from Kistwar, Jammu and Kashmir. Authors are thankful to NCBS CIFF facility for their help in flowcytometry analysis. SA is thankful to DST-Women Scientist-A research grant (SR/WOS-A/LS-96-2018) for funding this research.

\section{References}

1. Alonge M, Shumate A, Puiu D, Zimin AV, Salzberg SL. 2020. Chromosome-Scale Assembly of the Bread Wheat Genome Reveals Thousands of Additional Gene Copies. Genetics. Oct;216(2):599-608. doi: 10.1534/genetics.120.303501. Epub 2020 Aug 12. PMID: 32796007; PMCID: PMC7536849.

2. Alsayied NF, Fernández JA, Schwarzacher T, Heslop-Harrison JS. 2015. Diversity and relationships of Crocus sativus and its relatives analysed by inter-retroelement amplified polymorphism (IRAP). Ann Bot. 2015 Sep;116(3):359-68. doi: 10.1093/aob/mcv103. Epub 2015 Jul 1. PMID: 26138822; PMCID: PMC4549961.

3. Andrews S. 2010. FastQC: A Quality Control Tool for High Throughput Sequence Data. Available online: http://www.bioinformatics.babraham.ac.uk/projects/fastqc

4. Baba SA, et al. 2015. Comprehensive transcriptome analysis of Crocus sativus for discovery and expression of genes involved in apocarotenoid biosynthesis. BMC Genomics 16, 698 (2015).

5. Beier S, Thiel T, Münch T, Scholz U, Mascher M. 2017. MISA-web: A web server for microsatellite prediction. Bioinformatics 2017, 33, 2583-2585

6. Bolger AM, Lohse M, Usadel B. Trimmomatic: a flexible trimmer for Illumina sequence data. Bioinforma Oxf Engl. 2014;30:2114-20.

7. Brandizzi F, Grilli Caiola M. 1996. Quantitative DNA analysis in different Crocus species (Iridaceae) by mean of flow cytometry. Giornale Bot. Italiano 130: 643-645.

8. Brandizzi F, Grilli Caiola M. 1998. Flow cytometric analysis of nuclear DNA in Crocus sativus and allies (Iridaceae). Plant Syst. Evol. 211:149-154.

9. Busconi M, Soffritti G, Stagnati L, Marocco L, Martínez JM, De Los Mozos Pascual M, Fernandez JA. 2018. Epigenetic stability in Saffron (Crocus sativus L.) accessions during four consecutive years of cultivation and vegetative propagation under open field conditions. Plant Sci., 277 (2018), pp. 1-10

10. Campbell MS, Holt C, Moore B, Yandell M. 2014. Genome annotation and curation using MAKER and MAKER-P. Curr Protoc Bioinforma. 2014;48:4.11.1-39.

11. Chikhi, R.; Medvedev, P. 2014. Informed and automated k-mer size selection for genome assembly. Bioinformatics, 30, 31-37. 
12. Choi, J.Y, Lye, Z.N, Groen, S.C. et al. 2020. Nanopore sequencing-based genome assembly and evolutionary genomics of circum-basmati rice. Genome Biol 21, 21 (2020). https:// doi.org/10.1186/s13059-020-1938-2

13. David M. Goodstein, Shengqiang Shu, Russell Howson, Rochak Neupane, Richard D. Hayes, Joni Fazo, Therese Mitros, William Dirks, Uffe Hellsten, Nicholas Putnam, and Daniel S. Rokhsar. 2012. Phytozome: a comparative platform for green plant genomics, $\mathrm{Nu}$ cleic Acids Res. 40 (D1): D1178-D1186

14. Edwards, D, Batley, J. 2010. Plant genome sequencing: applications for plant improvement. Plant Biotech. J. 8: 2-9.

15. Feng S, He R, Lu J, Jiang M, Shen X, Jiang Y, Wang Z and Wang H. 2016. Development of SSR Markers and Assessment of Genetic Diversity in Medicinal Chrysanthemum morifolium Cultivars. Front. Genet. 7:113. doi: 10.3389/fgene.2016.00113

16. Hare EE, Johnston JS. 2011. Genome size determination using flow cytometry of propidium iodide-stained nuclei. Methods Mol Biol. 772:3-12. doi: 10.1007/978-1-61779-228-1_1. PMID: 22065429.

17. Harkess A, Zhou J, Xu C. et al. 2017. The asparagus genome sheds light on the origin and evolution of a young Y chromosome. Nat Commun 8, 1279 (2017). https://doi.org/10.1038/ s41467-017-01064-8

18. Jain M, Srivastava P, Verma M. et al. 2016. De novo transcriptome assembly and comprehensive expression profiling in Crocus sativus to gain insights into apocarotenoid biosynthesis. Sci Rep 6, 22456. https://doi.org/10.1038/srep22456

19. Jia Ye, Yong Zhang, Huihai Cui, Jiawei Liu, Yuqing Wu, Yun Cheng, Huixing Xu, Xingxin Huang, Shengting Li, An Zhou, Xiuqing Zhang, Lars Bolund, Qiang Chen, Jian Wang, Huanming Yang, Lin Fang, Chunmei Shi. 2018. WEGO 2.0: a web tool for analyzing and plotting GO annotations, 2018 update, Nucleic Acids Research, Volume 46, Issue W1, 2 July 2018, Pages W71-W75, https://doi.org/10.1093/nar/gky400

20. Jinpu Jin, Feng Tian, De-Chang Yang, Yu-Qi Meng, Lei Kong, Jingchu Luo, Ge Gao. 2017. PlantTFDB 4.0: toward a central hub for transcription factors and regulatory interactions in plants, Nucleic Acids Research, Volume 45, Issue D1, January 2017, Pages D1040-D1045, https://doi.org/10.1093/nar/gkw982

21. Langmead, B. 2010. Aligning short sequencing reads with Bowtie. Curr. Protoc. Bioinforma. 32, 11.7.1-11.7.14.

22. Li H, Durbin R. 2010. Fast and accurate long-read alignment with burrows-wheeler transform. Bioinformatics. 26(5):589-95. 
23. Liu, J, Seetharam, A.S, Chougule, K. et al. 2020. Gapless assembly of maize chromosomes using long-read technologies. Genome Biol 21, 121. https://doi.org/10.1186/s13059-020$\underline{02029-9}$

24. Luo, R.; Liu, B.; Xie, Y.; Li, Z.; Huang, W.; Yuan, J.; Wang, J. 2012. SOAPdenovo2: An empirically improved memory-efficient short-read de novo assembler. Gigascience 2012, 1, 18.

25. Maggi MA, Bisti S and Picco C. 2020. Saffron: Chemical Composition and Neuroprotective Activity, Molecules 2020, 25, 5618. doi:10.3390/molecules25235618.

26. Magotra S, Bhagat N, Ambardar S. et al. 2021. Field evaluation of PGP Bacillus sp. strain D5 native to Crocus sativus, in traditional and non traditional areas, and mining of PGP genes from its genome. Sci Rep 11, 5454. https://doi.org/10.1038/s41598-021-84585-z.

27. Marçais, G, and Kingsford, C. 2011. A fast, lock-free approach for efficient parallel counting of occurrences of k-mers. Bioinformatics 27, 764-770. doi: 10.1093/bioinformatics/btr011

28. Mir MA, Mansoor S,Sugapriya M, Alyemeni MN, Wijaya L, Ahmad P. 2021. Deciphering genetic diversity analysis of saffron (Crocus sativus L.) using RAPD and ISSR markers. Saudi Journal of Biological Sciences.28( 2) : 1308-1317

29. Moriya Y, Itoh M, Okuda S, Yoshizawa AC, Kanehisa M. 2007. KAAS: an automatic genome annotation and pathway reconstruction server. Nucleic Acids Res. 2007 Jul;35(Web Server issue):W182-5. doi: 10.1093/nar/gkm321. PMID: 17526522; PMCID: PMC1933193.

30. Mzabri I, Addi M, Berrichi A. 2019. Traditional and Modern Uses of Saffron (Crocus Sativus). Cosmetics.6(4):63. https://doi.org/10.3390/cosmetics6040063

31. Nemati Z, Harpke D, Gemicioglu A, Kerndorff H, Blattner F.R. 2019. Saffron (Crocus sativus) is an autotriploid that evolved in Attica (Greece) from wild Crocus cartwrightianus. Mol Phylogenet Evol, 136, pp. 14-20

32. Ranallo-Benavidez TR, Jaron KS, Schatz MC. 2020. GenomeScope 2.0 and Smudgeplot for reference-free profiling of polyploid genomes. Nat Commun. 11(1):1432. Published 2020 Mar 18. doi:10.1038/s41467-020-14998-3

33. Rogers S. O. and Bendich A. J. 1994. Extraction of total cellular DNA from plants, algae and fungi. In Plant molecular biology manual. (ed. S. B. Gelvin and R. A. Schilperoort), 2nd edition, vol. D1, pp. 1-8. Kluwer Academic Press, Dordrecht.

34. Rubio-Moraga A, Castillo-López R, Gómez-Gómez R, Ahrazem O. 2009. Saffron is a monomorphic species as revealed by RAPD, ISSR and microsatellite analyses. BMC Res. Notes, 2 (1), p. 189, 10.1186/1756-0500-2-189 
35. Samarghandian S, Borji A. 2014. Anticarcinogenic effect of saffron (Crocus sativus L.) and its ingredients. Pharmacogn. Res. 6:99-107. doi: 10.4103/0974-8490.128963.

36. Simão, F.A.;Waterhouse, R.M.; Ioannidis, P.; Kriventseva, E.V.; Zdobnov, E.M. 2015. BUSCO: Assessing genome assembly and annotation completeness with single-copy orthologs. Bioinformatics 2015, 31, 3210-3212.

37. Siracusa, L, Gresta, F, Avola, G, Albertini, E, Raggi, L, Marconi, G, et al. 2013. Agronomic, chemical and genetic variability of saffron (Crocus sativus L.) of different origin by LCUV-vis-DAD and AFLP analyses. Genet. Resour. Crop Evol. 60, 711-721. doi: 10.1007/ s10722-012-9868-9

38. Smit, A.F.A.; Hubley, R.; Green, P. 2010. RepeatMasker Open-4.0.. Available online: http://www.repeatmasker.org

39. Wang Z, Hobson N, Galindo L, et al. 2012. The genome of flax (Linum usitatissimum. L) assembled de novo from short shotgun sequence reads. Plant J. 72(3):461-473.

40. Xia E, Tong W, Hou Y, An Y, Chen L, Wu Q, Liu Y, Yu J, Li F, Li R, et al. 2020. The reference genome of tea plant and resequencing of 81 diverse accessions provide insights into genome evolution and adaptation of tea plants. Mol Plant. 13(7):1013-1026.

41. Xu L, Dong Z, Fang L, Luo Y, Wei Z, Guo H, Zhang G, Gu YQ, Coleman-Derr D, Xia Q, Wang Y. 2019. OrthoVenn2: a web server for whole-genome comparison and annotation of orthologous clusters across multiple species. Nucleic Acids Res. 2019 Jul 2;47(W1):W52W58. doi: 10.1093/nar/gkz333. PMID: 31053848; PMCID: PMC6602458.

42. Zhang YH, Zheng LL, Zheng Y, Zhou C, Huang P, Xiao X, et al. 2019. Assembly and annotation of a draft genome of the medicinal plant Polygonum cuspidatum. Front Plant Sci. 10:1274. https://doi.org/10.3389/fpls.2019.01274.

43. Zimin AV, Marçais G, Puiu D, Roberts M, Salzberg SL, Yorke JA. 2013. The MaSuRCA genome assembler. Bioinformatics. 2013; 29(21):2669-77.

\section{Table legends:}

Table 1: Total raw data of 321.36 Gigabases obtained from two insert size (500 bp and $800 \mathrm{bp}$ ) libraries using Illumina sequencing with an overall coverage of $\sim 92 X$. The genome size was estimated as 3.5 Gigabases $(3,578,575,507$ bases $)$.

Table 2: Assembly statistics of C. sativus genome using soapdenovo2 and MaSuRCA de-novo assemblers.

Table 3: Mapping WGS raw reads and previous published transcriptome data to Cs_assembly_2. 
341 Table 4: Classification of repetitive sequences in $C$. sativus genome representing abundance of 342 Simple repeats and LTRs.

343 Table 5: SSR markers from Crocus sativus draft genome (Cs_assembly_2) depicting the more rela344 tive abundance of monomeric repeat microsatellite.

345 Table 6: Number of genes annotated against NR and Viridiplantaea database depicting more num346 ber of proteins annotated against Viridiplantaea database.

\section{Figure legends:}

348 Fig 1: Schematic of de novo genome assembly and annotation pipeline. Black colour text represent 349 the analytical processes and Red colour text represent the software/instrument used to perform the 350 processes.

351 Fig 2: Crocus sativus unigenes mapping to top 15 plant species wherein most of the proteins anno352 tated against Asparagus officinalis.

353 Fig 3: Trancription factors identified in Crocus sativus genome wherein MYB \& MYB related Tfs 354 were most abundant.

355 Fig 4a: Orthology analysis of Crocus sativus with neighbouring plants from same order along with 356 Rice representing 7328 proteins clusters to be conserved in all the five plant species, whereas 2510 357 proteins cluster were unique to $C$. sativus only

358 Fig 4b: Overlapping cluster numbers between each pair of plant species representing common clusters (7328) among five plant species and unique cluster (2510) to Crocus sativus.

360 Fig 5: Heatmap of overlapping cluster numbers between each pair of plant species representing more number of overlapping clusters between Crocus sativus and Asparagus officinalis.

362 Fig 6: Carotene biosynthesis pathway in Crocus sativus that was found complete with all the genes 363 in the pathway. 

(which was not certified by peer review) is the author/funder. All rights reserved. No reuse allowed without permission.

369 Table 1: Total raw data of 321.36 Gigabases obtained from two insert size (500 bp and $800 \mathrm{bp}$ ) li-

370 braries using Illumina sequencing with an overall coverage of $\sim 92 \mathrm{X}$. The genome size was esti-

371 mated as 3.5 Gigabases $(3,578,575,507$ bases $)$.

372

\begin{tabular}{|l|l|c|l|l|}
\hline \multirow{3}{*}{ Libraries } & Sample Name & $\begin{array}{c}\% \\
\text { GC }\end{array}$ & $\begin{array}{c}\text { Sequences } \\
\text { (Million } \\
\text { Read) }\end{array}$ & $\begin{array}{c}\text { Data } \\
\text { (in Giga- } \\
\text { bases) }\end{array}$ \\
\hline \multirow{3}{*}{800} & Lib1_800_R1 & $46 \%$ & 93.2 & 13.98 \\
\cline { 2 - 5 } & Lib1_800_R2 & $46 \%$ & 93.2 & 13.98 \\
\cline { 2 - 5 } & Lib2_800_R1 & $46 \%$ & 357.8 & 53.68 \\
\cline { 2 - 5 } & Lib2_800_R2 & $46 \%$ & 357.8 & 53.68 \\
\hline \multirow{3}{*}{500} & Lib3_500_R1 & $46 \%$ & 300.1 & 45.02 \\
\cline { 2 - 5 } & Lib3_500_R2 & $46 \%$ & 300.1 & 45.02 \\
\cline { 2 - 5 } & Lib4_500_R1 & $46 \%$ & 320.0 & 48.01 \\
\cline { 2 - 5 } & Lib4_500_R2 & $46 \%$ & 320.0 & 48.01 \\
\hline \multirow{2}{*}{ Total } & & & $\mathbf{2 1 4 2 . 2}$ & $\mathbf{3 2 1 . 3 6}$ \\
\hline
\end{tabular}

374 Table 2: Assembly statistics of C. sativus genome using soapdenovo2 and MaSuRCA de-novo as375 semblers.

\begin{tabular}{|l|l|l|l|l|}
\hline & \multicolumn{3}{|l|}{ Soapdenovo2 } & MaSuRCA \\
\hline Assemblies & - & Cs_Assembly_1 & - & $\begin{array}{l}\text { Cs_Assem- } \\
\text { bly_2 }\end{array}$ \\
\hline kmers & 69 & $\mathbf{7 1}$ & 73 & 99 \\
\hline N50 Scaffold (bases) & 1443 & $\mathbf{1 5 9 6}$ & 1508 & $\mathbf{1 8 6 3}$ \\
\hline Number of Scaffolds & 1537310 & $\mathbf{1 5 0 5 1 2 9}$ & 1433675 & $\mathbf{2 5 6 4 0 4 2}$ \\
\hline Largest Scaffold (bases) & 45973 & $\mathbf{4 5 9 7 3}$ & 43370 & $\mathbf{4 6 7 3 4}$ \\
\hline Total sequence length & 2684437407 & $\mathbf{2 7 8 7 9 2 6 2 8 0}$ & 2589039086 & $\mathbf{3 0 1 4 6 1 2 5 6 3}$ \\
\hline GC\% & 43.2 & $\mathbf{4 3 . 2}$ & 43.2 & $\mathbf{4 3 . 2}$ \\
\hline Genome Coverage (\%) & $75.01 \%$ & $\mathbf{7 7 . 9 0 \%}$ & $72.34 \%$ & $\mathbf{8 4 . 2 4 \%}$ \\
\hline BUSCO (\%) & $7.32 \%$ & $\mathbf{7 . 8 1 \%}$ & $7.05 \%$ & $\mathbf{4 4 . 4 6 \%}$ \\
\hline
\end{tabular}


25 bioRxiv preprint doi: https://doi.org/10.1101/2021.06.23.449592; this version posted June 23, 2021. The copyright holder for this preprint (which was not certified by peer review) is the author/funder. All rights reserved. No reuse allowed without permission.

378 Table 3: Mapping WGS raw reads and previous published transcriptome data to Cs_assembly_2.

\begin{tabular}{|l|l|l|l|}
\hline Data Type & $\begin{array}{l}\text { WGS raw reads } \\
\text { in present study }\end{array}$ & $\begin{array}{l}\text { C.sativus } \\
\text { transcripts } \\
\text { (Jain et al., 2016) }\end{array}$ & $\begin{array}{l}\text { C.sativus } \\
\text { transcriptome raw reads } \\
\text { (Baba et al 2015) }\end{array}$ \\
\hline Source of Data & Present study & Jain et al., 2016 & Baba et al 2015 \\
\hline $\begin{array}{l}\text { Total number of reads/tran- } \\
\text { scripts }\end{array}$ & 2135957246 & 327920 & 59043670 \\
\hline Mapped reads/transcripts & 1864292472 & 327643 & 54330850 \\
\hline Mapping Percentage & $87.28 \%$ & $99.92 \%$ & $92.02 \%$ \\
\hline
\end{tabular}

382 Table 4: Classification of repetitive sequences in C. sativus genome representing abundance of 383 Simple repeats and LTRs.

\begin{tabular}{|l|l|}
\hline Repetitive region & Numbers \\
\hline Simple repeats & 415561 \\
\hline LTR & 260472 \\
\hline Low_complexity & 64624 \\
\hline DNA & 64205 \\
\hline LINE & 45739 \\
\hline Satellite & 3946 \\
\hline RC_Helitron & 3072 \\
\hline rRNA & 2749 \\
\hline SINE & 848 \\
\hline tRNA & 650 \\
\hline Other & 340 \\
\hline snRNA & 54 \\
\hline Retroposon & 15 \\
\hline Total & $\mathbf{8 6 2 2 7 5}$ \\
\hline
\end{tabular}


27 bioRxiv preprint doi: https://doi.org/10.1101/2021.06.23.449592; this version posted June 23, 2021. The copyright holder for this preprint (which was not certified by peer review) is the author/funder. All rights reserved. No reuse allowed without permission.

388 Table 5: SSR markers from Crocus sativus draft genome (Cs_assembly_2) depicting the more rela389 tive abundance of monomeric repeat microsatellite.

\begin{tabular}{|l|l|l|l|l|}
\hline SSR types & count & $\begin{array}{l}\text { relative } \\
\% \text { age }\end{array}$ & $\begin{array}{l}\text { Most abun- } \\
\text { dant }\end{array}$ & \%age \\
\hline monomeric repeat microsatellite & 486140 & $50.4 \%$ & "A" & $44.6 \%$ \\
\hline dinucleotide repeat microsatellite & 294819 & $30.5 \%$ & "TA" & $16.5 \%$ \\
\hline trinucleotide repeat microsatellite & 146991 & $15.2 \%$ & "TTG" & $5.72 \%$ \\
\hline Tetranucleotide repeat microsatellite & 15375 & $1.59 \%$ & "AAAT" & $7.7 \%$ \\
\hline Pentanucleotide repeat microsatellite & 8596 & $0.9 \%$ & "TATAT" & $3.2 \%$ \\
\hline Hexanucleotide repeat microsatellite & 12310 & $1.27 \%$ & "TAACCC" & $5.3 \%$ \\
\hline Total & $\mathbf{9 6 4 2 3 1}$ & & & \\
\hline
\end{tabular}

392 Table 6: Number of genes annotated against NR and Viridiplantaea database depicting more num393 ber of proteins annotated against Viridiplantaea database.

\begin{tabular}{|l|l|l|l|l|}
\hline Databases & $\begin{array}{l}\text { Total maker } \\
\text { annotated }\end{array}$ & $\begin{array}{l}\text { Total annotated } \\
\text { proteins }\end{array}$ & $\begin{array}{l}\mathbf{> 4 0} \text { \% percentage iden- } \\
\text { tity } \& \mathbf{7 0 \%} \text { query cov- } \\
\text { erage }\end{array}$ & $\begin{array}{l}\text { Unique accession } \\
\text { numbers }\end{array}$ \\
\hline Viridiplantaea & 254038 & 146118 & 107553 & 52546 \\
\hline NR & 254038 & 143745 & 107385 & 52436 \\
\hline
\end{tabular}




\section{Figures:}

404

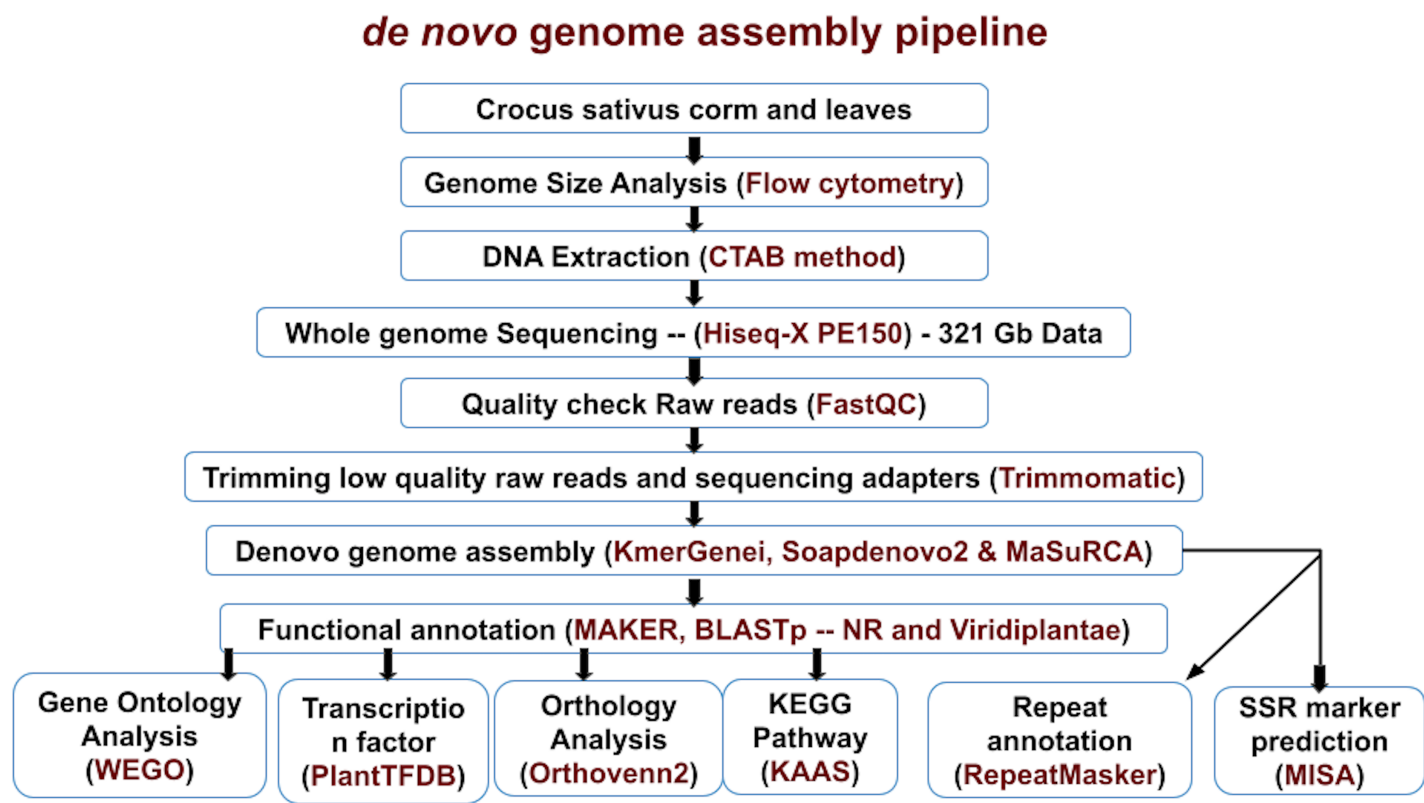

406 Fig 1: Schematic of de novo genome assembly and annotation pipeline. Black colour text represent

407 the analytical processes and Red colour text represent the software/instrument used to perform the 408 processes.

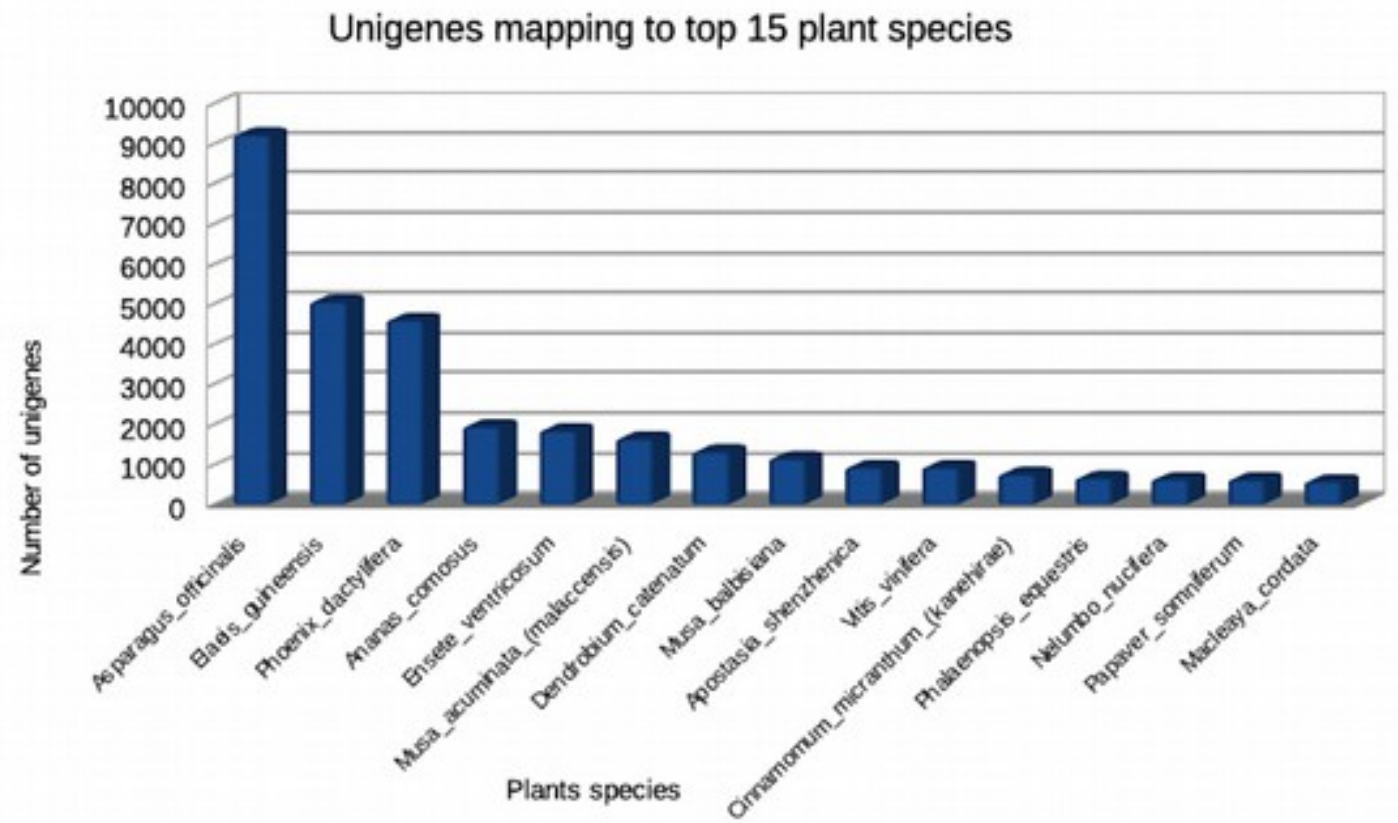

410 Fig 2: Crocus sativus unigenes mapping to top 15 plant species wherein most of the proteins anno411 tated against Asparagus officinalis. 
31 bioRxiv preprint doi: https://doi.org/10.1101/2021.06.23.449592; this version posted June 23, 2021. The copyright holder for this preprint (which was not certified by peer review) is the author/funder. All rights reserved. No reuse allowed without permission.

\section{Transcription factors in C.sativus genome}

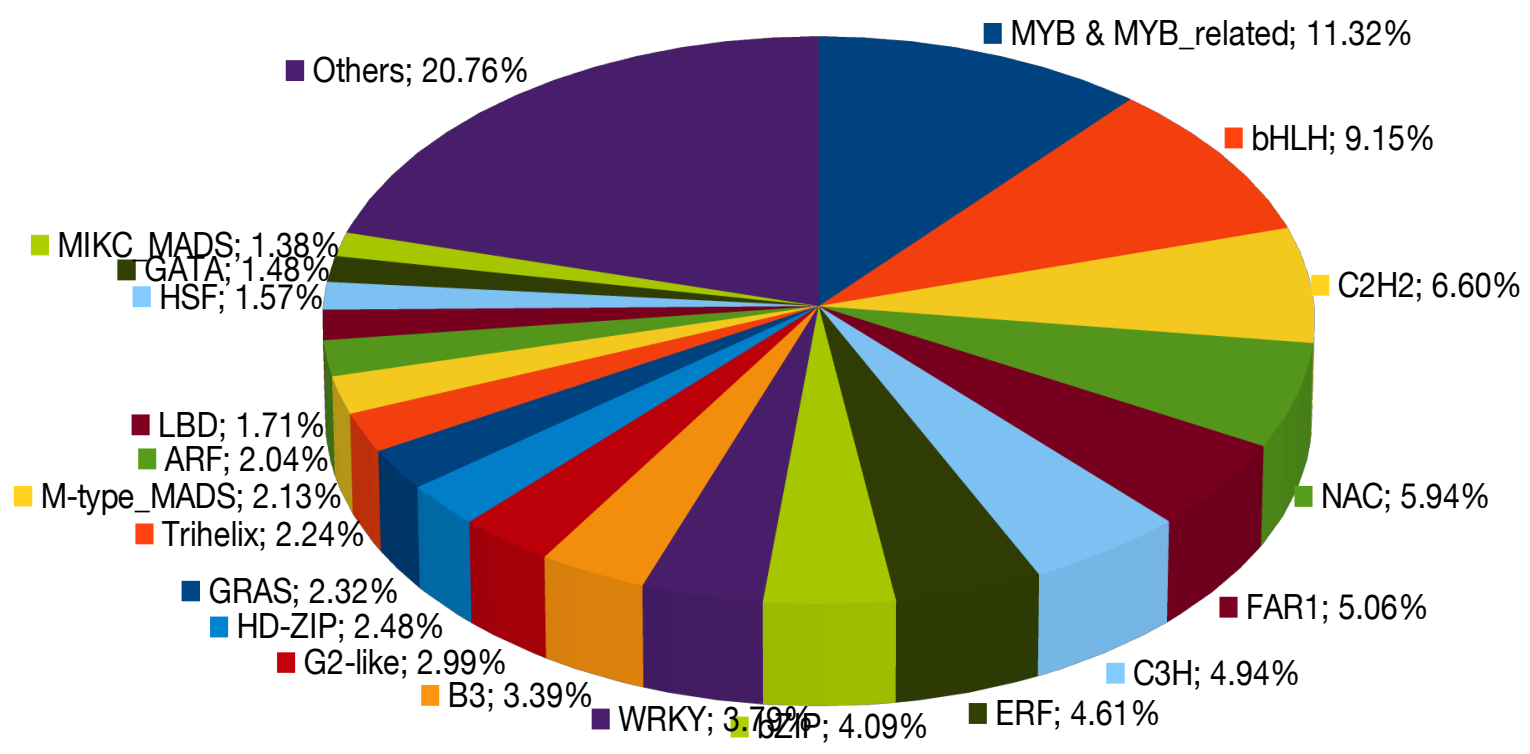

418 Fig 3: Trancription factors identified in Crocus sativus genome wherein MYB \& MYB related Tfs 419 were most abundant. 

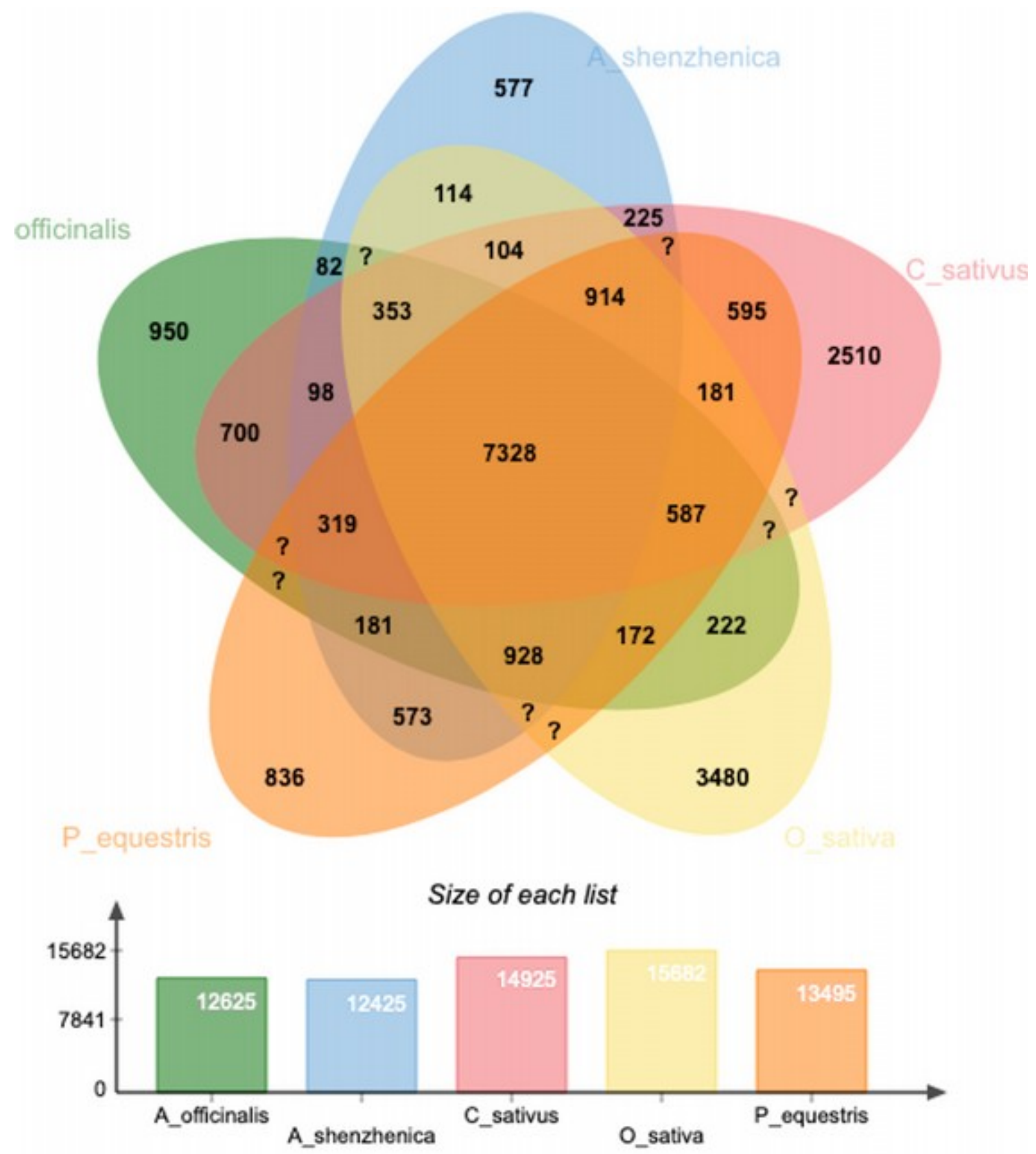

Number of elements: specific (1) or shared by 2, 3, ... lists

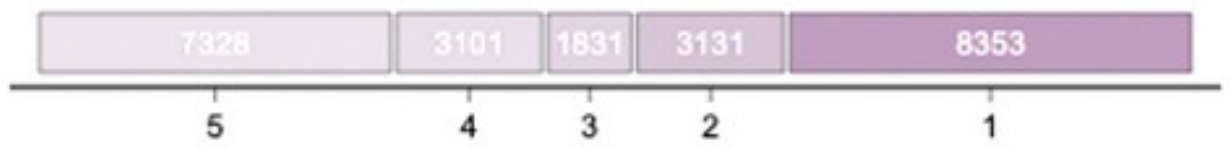

425 Fig 4a: Orthology analysis of Crocus sativus with neighbouring plants from same order along with 426 Rice representing 7328 proteins clusters to be conserved in all the five plant species, whereas 2510 427 proteins cluster were unique to $C$. sativus only. 


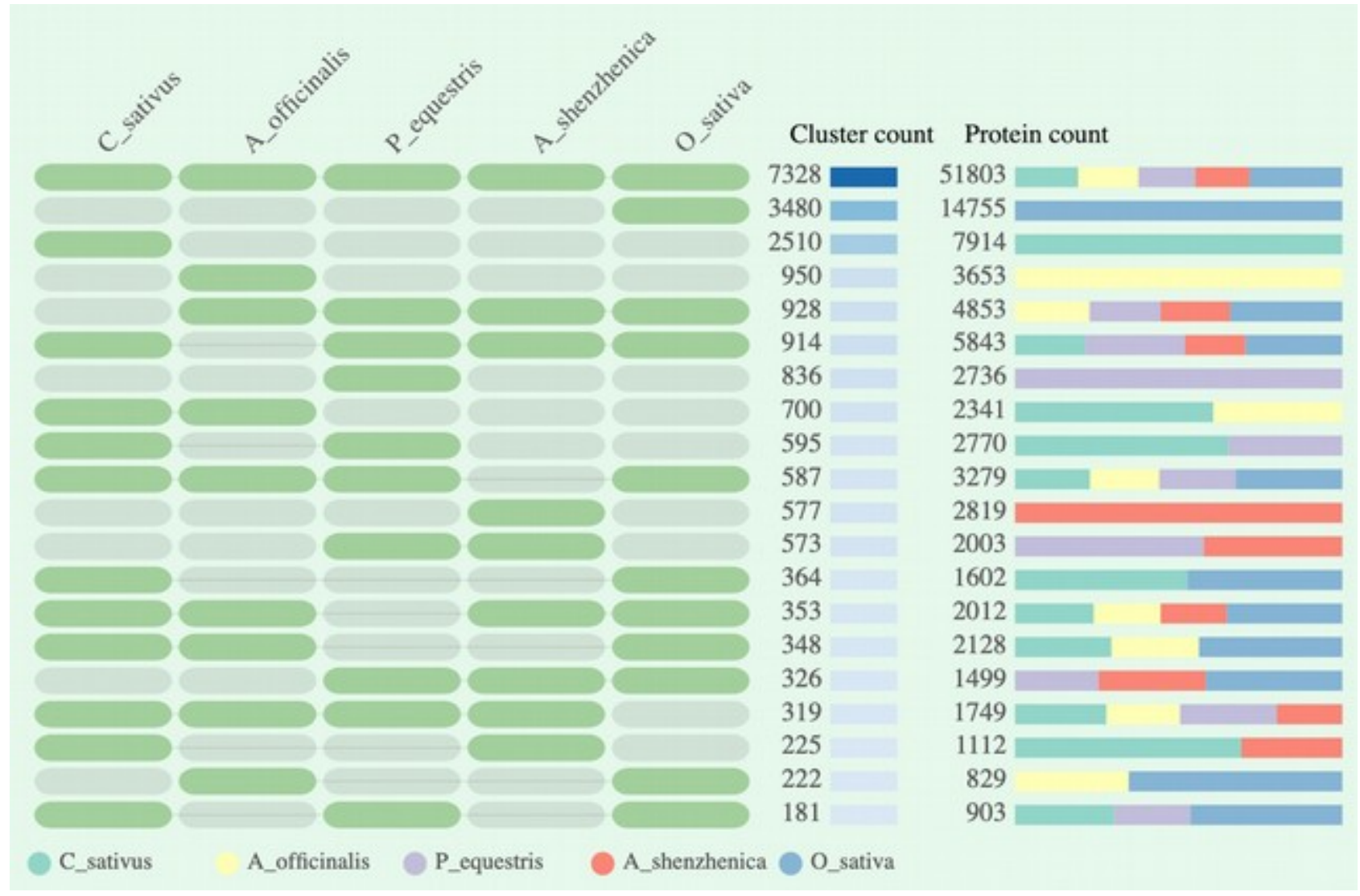

430 Fig 4b: Overlapping cluster numbers between each pair of plant species representing common clus431 ters (7328) among five plant species and unique cluster (2510) to Crocus sativus. 
37 bioRxiv preprint doi: https://doi.org/10.1101/2021.06.23.449592; this version posted June 23, 2021. The copyright holder for this preprint (which was not certified by peer review) is the author/funder. All rights reserved. No reuse allowed without permission.

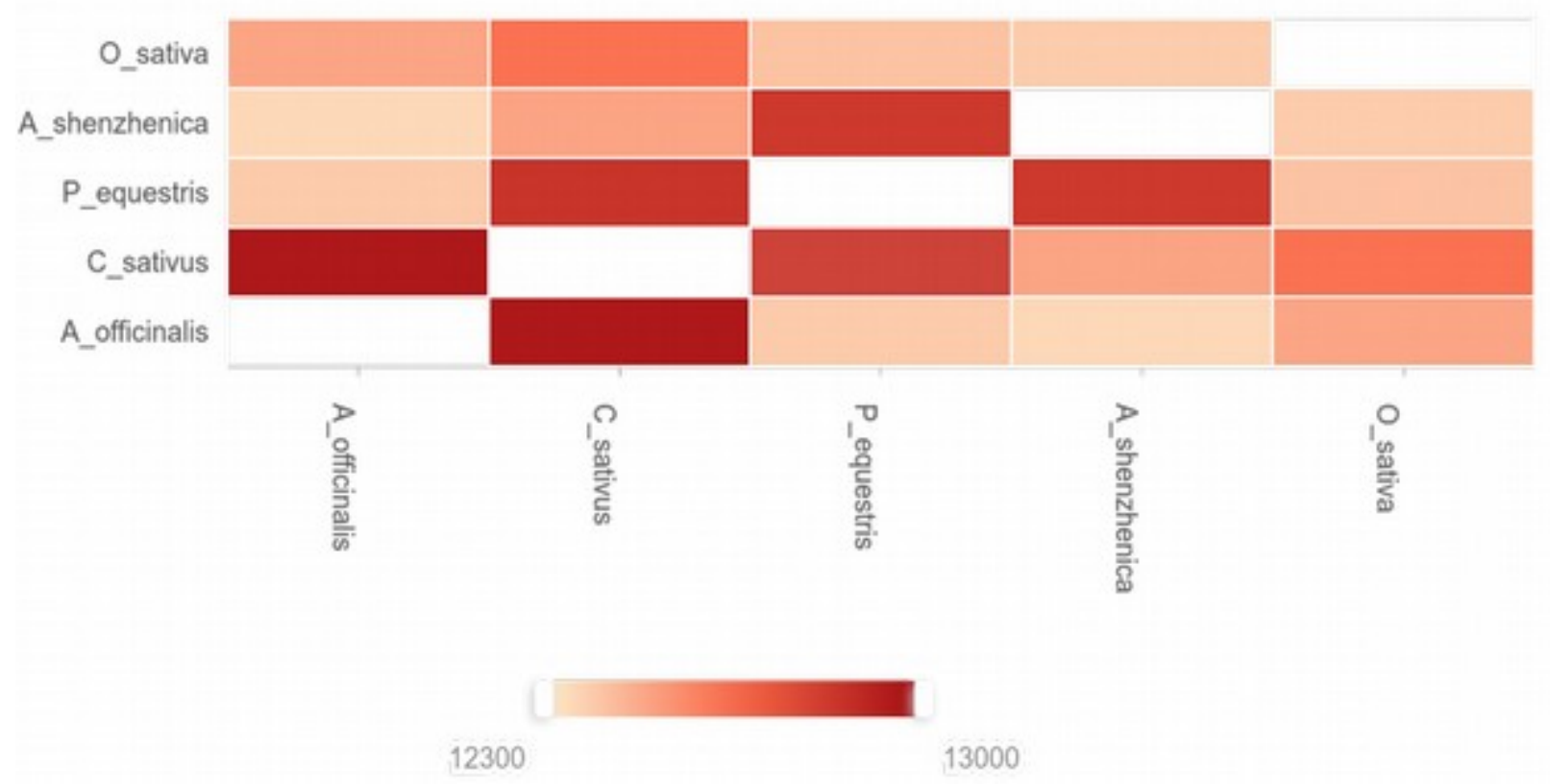

444

445 Fig 5: Heatmap of overlapping cluster numbers between each pair of plant species representing 446 more number of overlapping clusters between Crocus sativus and Asparagus officinalis. 


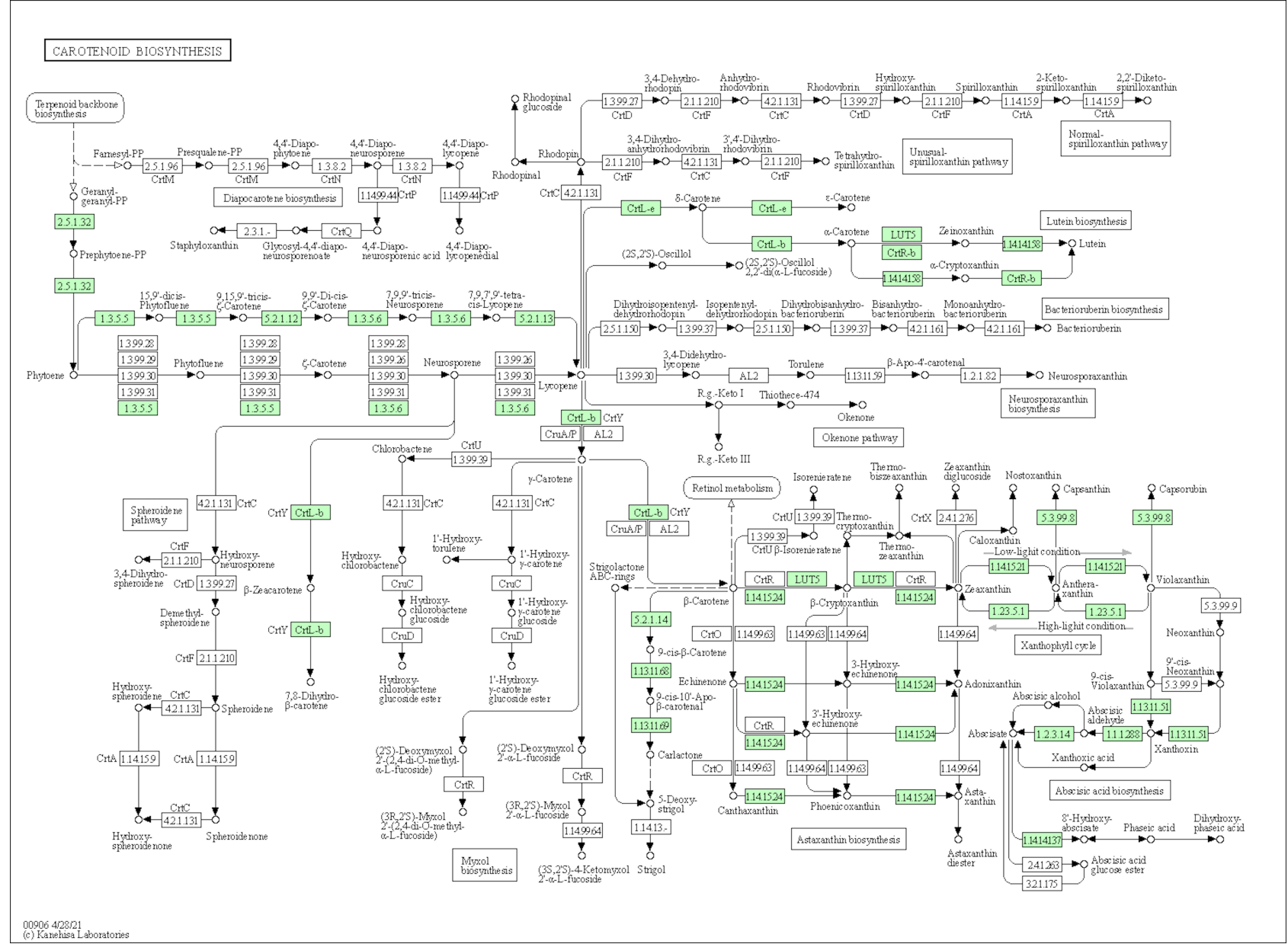

457 Fig 6: Carotene biosynthesis pathway in Crocus sativus that was found complete with all the genes 458 in the pathway. 